\title{
Automated and Accurate Measurement of Aortic Pulse Wave Velocity Using Magnetic Resonance Imaging
}

\author{
SS Giri, Y Ding, Y Nishijima, A Pedraza-Toscano, \\ PM Burns, RL Hamlin, OP Simonetti \\ The Ohio State University, Columbus, OH, USA
}

\begin{abstract}
Arterial stiffness is thought to be a powerful indicator of present and possible future cardiovascular risk. Accordingly, there is an increasing interest in the non-invasive clinical measurement of arterial stiffness. The commonly used non-invasive methods assess aortic Pulse Wave Velocity (PWV) which is known to be a good indicator of aortic stiffness. These methods calculate the $P W V$ by measuring the pressure or flow velocity at two locations along the aorta to determine the arrival time of the pulse wave; the distance between these locations is measured either on the body surface or using high resolution images. In this study, we have developed a novel method based on MRI that calculates the aortic $P W V$ using multiple locations along the aorta. This approach is largely automatic vis-a-vis aortic segmentation that reduces user-subjectivity; the inclusion of multiple points along aorta increases the accuracy of $P W V$ measurement by averaging.
\end{abstract}

\section{Introduction}

The heart pumps blood in a pulsatile fashion, generating pressure waves that propagate through the aorta and the peripheral arteries at a rate termed the Pulse Wave Velocity (PWV) [1]. Aortic PWV is widely accepted as an excellent target parameter for the assessment of aortic stiffness which is known to be an independent marker of cardiovascular morbidity and mortality: Meaume et al. [2] studied the relation between aortic PWV and cardiovascular mortality in subjects above 70 years of age. Lim et al. [3] showed that Aortic PWV is an independent marker of Coronary Artery Disease. Thus, there is an increasing interest in the clinical measurement of arterial stiffness.

Current methods used to determine PWV include invasive catheter-based measurements, and non-invasive methods based on applanation tonometry, Doppler and MRI. Tonometry records the pressure waveforms at the carotid and femoral arteries; distance between these 2 sites is measured on the surface [4]. This approach (tonometry) suffers from 2 drawbacks

1. Carotid and femoral PWV may differ from that in the aorta. This may degrade the accuracy of aortic PWV estimates by this method.

2. The surface distances are approximate, especially in cases where the central artery is tortuous.

The Doppler method [5] rectifies the first of these problems but fails to address the second.

MRI overcomes this by providing large FOV, high resolution anatomical images in any plane. Phase Contrast MRI (PCMRI) [6] also gives an accurate assessment of the flow velocity.

Previous MRI studies have attempted to measure PWV by plotting velocity curves at a few predefined points along the aorta $[7,8]$. The arrival time of the pulse wave at these locations was determined from the velocity curves. The distance between these locations was measured using high resolution anatomical images in a para-sagittal plane. PWV was then measured as

$$
\mathrm{PWV}=\Delta \mathrm{d} / \Delta \mathrm{t}
$$

We have developed a novel approach based on a single para-sagittal phase-velocity map of the aorta. These images include the aorta from the heart to the abdominal region just above its bifurcation. The blood flow velocity is measured at a multitude of pixels throughout the aorta, providing the necessary data for accurate estimation of aortic PWV.

\section{Methods}

\section{i. Image Acquisition}

Images were acquired in a Siemens Magnetom 1.5T MRI scanner (Avanto, Siemens, Erlangen, Germany). A spoiled Gradient Echo (GRE) sequence was used with a flip angle of 15 degrees and retrospective Electrocardiographic (ECG) gating to acquire a "candycane" view of the thoracic and abdominal (Fig 1). The FOV was $340 \times 255 \mathrm{~mm}$ with a spatial resolution of $1.5 \mathrm{~mm} \times 1.5 \mathrm{~mm} \times 5 \mathrm{~mm}$. The Echo Time (TE) was $3 \mathrm{~ms}$ and the repetition time (TR) $14 \mathrm{~ms}$. PCMRI used a Venc of $\pm 150 \mathrm{~cm} / \mathrm{s}$. The velocity encoding direction was chosen to be head to foot. While the magnitude of the 
flow velocity may be underestimated where the flow is not directly along the head-foot direction, the amplitude of the velocity is not critical to accurate determination of the pulse wave arrival time.

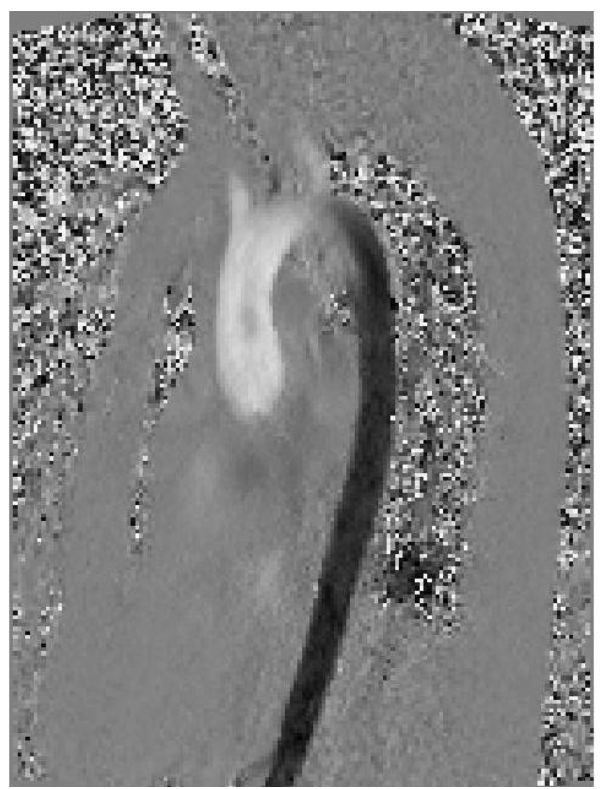

Figure 1. Para-sagittal slice showing Ascending and descending aorta

\section{ii. Post-processing}

All the images were processed using a GUI tool developed specifically for calculating PWV. This tool was built on MATLAB (The Mathworks, Natick, MA, USA).

The first step is to segment the aorta from the rest of the image. To do this, we designed and implemented an algorithm that correlates the changes in the intensity of a single user-defined aortic pixel with those of rest of the pixels across the cardiac cycle. Thus, if the user selects a point in the aorta, all the points in the aorta will have a high correlation with the selected point due to the blood flow in aorta. The rest of the background will have low correlation with the selected point. In this way, we segment the aorta from the rest of the background. Some morphological operations may be needed to remove the regions of heart that may have similar correlation due to the blood pool in heart. The final segmented aorta is shown in fig 2.

After segmenting the aorta, we erode it from the sides until we get a line that represents the central line (Fig 3). If the aorta segmentation is not perfect, then there may be branches in the aorta central line. To eliminate the branches, we use another morphological operation called 'spur' in which we progressively trim the edges of the central line until there are no branches.

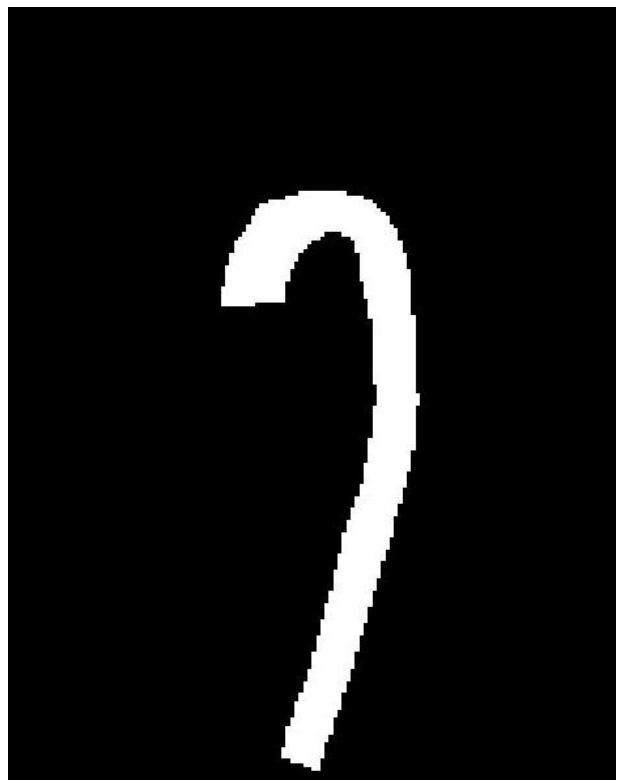

Figure 2. Segmented aorta.

We use this central line to calculate distance along the aorta. This is done using the pixel dimensions and simple geometry.

The aortic central line is also used to locate cross sections of aorta. This is done by associating each pixel of the central line with a cross section. Then, each pixel of aorta is compared with the pixels of central line. A pixel of aorta is assigned to the cross section whose central pixel is closest. We thus generate a series of aortic cross sections as demonstrated in Fig 4. The number of cross sections depends on the image resolution. At each cross section we use the PCMRI images to plot the vertical component of blood flow velocity throughout the cardiac cycle. Figure 5 shows the plot of the velocity curve taken at one cross-section. Similar plots are obtained for each of the cross sections.

The velocity curve is automatically analyzed at each cross section and the time of maximum jump in the leading edge is defined as the pulse wave arrival time.

This process is automated and is executed for every cross section. The use of the vertical component of velocity is justified because we are interested only in the time of arrival of pulse, which is not affected by a scaling factor (cosine of the angle between the actual flow velocity and vertical).

Thus, knowing the arrival time of the pulse wave at each of the cross-sections and the distance of each crosssection along the aorta, we can calculate the PWV using the simple distance/time formula. This is done as follows:

The distance of each cross-section relative to the first cross-section (which is taken as zero) is plotted against the arrival time of the pulse wave at that cross section. 


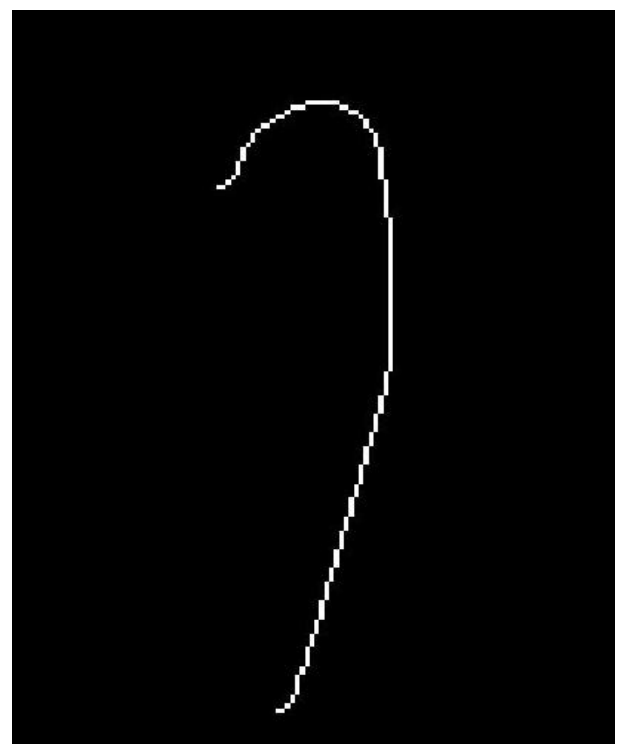

Fig 3 Central line of Aorta

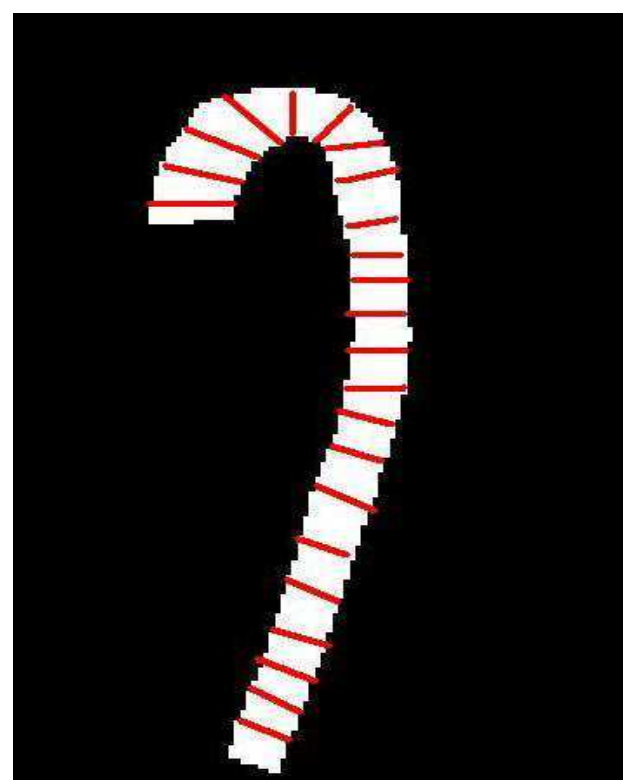

Fig 4 Some of the cross sections along the aorta. Only a few cross sections are shown for demonstration purpose. The algorithm computes a continuum of cross sections depending on the image resolution. This step is transparent to the user.

The slope of a linear regression fit of the distance vs. time data provides an estimate of the average PWV for the entire imaged section of aorta.

A non-zero intercept of the regression line is expected because distance is measured relative to the first cross section, while time is measured relative to the ECG $\mathrm{R}$-wave. Since only the slope is needed to estimate $\mathrm{PWV}$, the non-zero intercept can be ignored.

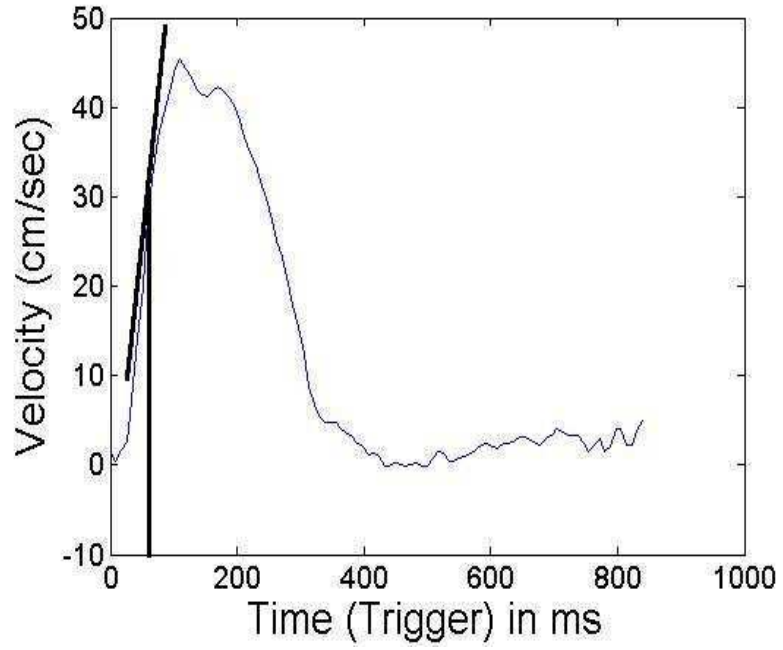

Fig 5: Velocity vs time waveform at a single cross section along the aorta. Also shown is a tangent drawn at the point where the velocity change is maximum. This point is taken as the arrival of the pulse and the corresponding time noted.

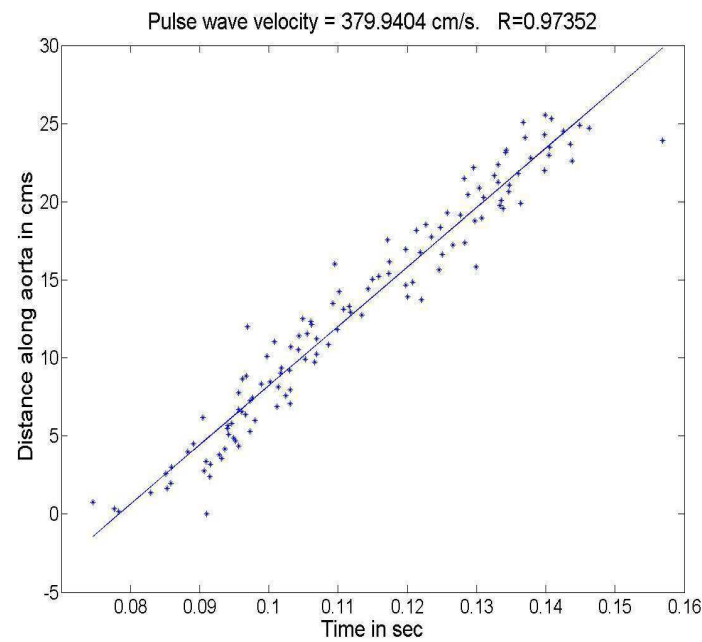

Fig 6: PWV is given by the slope of a linear regression fit of the distance vs. time data

\section{Results}

PWV of 3 dogs obtained from PC-MRI and the software tool were compared with those obtained from catheter based pressure wave measurements. Measurements were taken at baseline and during infusion of two drugs which altered aortic compliance. The results are summarized below in Table 1 . In each animal, an aortic length of more than $20 \mathrm{~cm}$ was imaged resulting in about 150 cross-sections along the aorta. The performance of the MRI method was validated using a catheter-based Millar solid-state pressure transducer. The 
values obtained with MRI showed a strong linear correlation with the catheter-based measurements $\left(\mathrm{R}^{2}=\right.$ 0.957).

\begin{tabular}{|c|c|c|c|}
\hline Subject & Type & Cath $(\mathrm{m} / \mathrm{s})$ & MRI $(\mathrm{m} / \mathrm{s})$ \\
\hline \multirow{3}{*}{ Dog 1} & Baseline & 4.41 & 3.04 \\
\cline { 2 - 4 } & Nitroprusside & 4.30 & 3.88 \\
\cline { 2 - 4 } & Phenylephrine & 3.95 & 3.92 \\
\hline \multirow{3}{*}{ Dog 2} & Baseline & 4.05 & 4.01 \\
\cline { 2 - 4 } & Nitroprusside & 4.29 & 4.02 \\
\cline { 2 - 4 } & Phenylephrine & 5.66 & 6.94 \\
\hline \multirow{3}{*}{ Dog 3 } & Baseline & 3.63 & 3.63 \\
\cline { 2 - 4 } & Nitroprusside & 3.65 & 3.72 \\
\cline { 2 - 4 } & Phenylephrine & 5.02 & 5.68 \\
\hline
\end{tabular}

Table 1: Comparison of PWV measured using a catheter and MRI. The two sets of values were correlated, with an $\mathrm{R}^{2}=$ 0.957. In each of these dogs, the PWV was computed over an aortic length of more than $20 \mathrm{cms}$, yielding about 150 crosssections for flow-velocity measurement.

\section{Discussion and conclusions}

A new method to measure the aortic Pulse Wave Velocity was developed. It directly measures distance along the aorta rather than relying on approximate body surface measurements. PC-MRI provides accurate flow velocity waveforms at multiple locations along the aorta in a single 2 minute scan, generating a large number of data points and an accurate estimate of PWV. This method also has the potential to estimate local PWV along the aorta to evaluate changes in local compliance that may be a precursor of aneurysm or other pathology.

A possible limitation of our method is that only a few pixels are used to compute the average velocity at each cross-section. More pixels would contribute to each velocity waveform if the image plane were oriented perpendicular, rather than parallel to the aorta $[7,8]$. While our method uses fewer points to generate the velocity waveform at any location, we measure the velocity at many more locations along the aorta than if a separate cross-sectional image had to be acquired for each. This greatly reduces data acquisition time, and potentially increases the accuracy of our measurement, as evidenced by the high correlation with invasive pressure wave measurements. This method also offers the possibility of estimating localized changes in aortic stiffness, which will be explored in future work.

\section{References}

[1] Milnor WR. Wave propagation. In: Hemodynamics. 2d ed. Baltimore: Williams \& Wilkins, 1989:225259

[2] Meaume S, Benetos A, Henry OF, Rudnichi A, Safar ME. Aortic pulse wave velocity predicts cardiovascular mortality in subjects $>70$ years of age. Arterioscler Thromb Vasc Biol 2001;21(12):204650.

[3] Lim HE, Park CG, Shin SH, Ahn JC, Seo HS, Oh DJ. Aortic pulse wave velocity as an independent marker of coronary artery disease. Blood Press 2004;13(6):369-75.

[4] Tsai PS, Yucha CB. Noninvasive measurements of central arterial pressure and distensibility by arterial applanation tonometry with a generalized transfer function: implications for nursing. Heart Lung 2001;30(6):437-44.

[5] Wright JS, Cruickshank JK, Kontis S, Dore C, Gosling RG. Aortic compliance measured by noninvasive Doppler ultrasound: description of a method and its reproducibility. Clin Sci (Lond) 1990;78(5):463-8.

[6] Lotz J, Meier C, Leppert A, Galanski M. Cardiovascular flow measurement with phasecontrast MR imaging: basic facts and implementation. Radiographics 2002;22(3):651-71.

[7] Rogers WJ, Hu YL, Coast D, Vido DA, Kramer CM, Pyeritz RE, et al. Age-associated changes in regional aortic pulse wave velocity. J Am Coll Cardiol 2001;38(4):1123-9.

[8] Gang G, Mark P, Cockshott P, Foster J, Martin T, Blyth K, et al. Measurement of pulse wave velocity using magnetic resonance imaging. Conf Proc IEEE Eng Med Biol Soc 2004;5:3684-7.

Address for correspondence

Shivraman Giri

Cardiac MRI Research Center

410 W. $10^{\text {th }}$ Ave.

Room 527 Doan Hall,

Columbus, $\mathrm{OH}-43210$ USA

E-mail: Shivraman.Giri@osumc.edu 